\title{
Bajo peso al nacer y su implicación en el desarrollo psicomotor
}

\author{
Doris Amparo Parada-Rico* \\ Nathalia López-Guerrero** \\ Marcela Martínez-Laverde ${ }^{* * *}$
}

ISSN 1794-9831

E-ISSN 2322-7028

Vol. 12 No. 2

Jul - Dic 2015

Cúcuta, Colombia

Recibido:

31 de Julio

de 2015

Aceptado por pares:

19 de Octubre de 2015

Aprobado:

26 de Noviembre

de 2015

*Enfermera

Magister en

Materno Perinatal.

Docente.

Universidad

Francisco de

Paula Santander.

Cúcuta, Colombia.

Correo electrónico:

dorisparada@ufps.

edu.co

**Enfermera

Enfermera Servicio

Social Obligatorio.

Hospital Jorge

Cristo Sahium,

Villa del Rosario,

Colombia. Correo

electrónico:

nathalialp4@gmail.

com

***Enfermera

Enfermera

Profesional

Programa

Materno Infantil

- IMSALUD.

Cúcuta, Colombia.

Correo electrónico:

leidymarcela2003@

hotmail.com

****Descriptores en Ciencias de la Salud (DeCS), en la página http://

decs.bvs.br/E/ homepagee.htm

de la Biblioteca

virtual en salud del proyecto BIREME,

de la Organización

Mundial de la

Salud y de la

Organización

Panamericana de

Salud. 


\section{Underweight birth and their involvement in the psychomotor development}

\section{ABSTRACT}

Children who are underweight at birth can develop serious health problems, so does the risk of problems and disabilities in their psychomotor development in the long term is greater. After the revision in scientific databases, is identified as a health problem worldwide, since these infants may have serious limitations in their survival and later in their quality of life; therefore, the psychomotor development of these children should thoroughly evaluate so that early detection of risk factors likely to ensure care interventions.

KEY WORDS: child development, psychomotor performance, underweight birth, underweight newborn. 


\section{O baixo peso ao nascimento e sua participação no desenvolvimento psicomotor}

ISSN 1794-9831 E-ISSN 2322-7028

Vol. 12 No. 2

Jul - Dic 2015

Cúcuta, Colombia

\section{RESUMO}

As crianças que estão abaixo do peso no nascimento podem desenvolver sérios problemas de saúde, assim como o risco de problemas e dificuldades no seu desenvolvimento psicomotor no longo prazo é maior. A revisão em bases de dados científicos, é identificada como um problema de saúde em todo o mundo, uma vez que estas crianças podem ter sérias limitações na sua sobrevivência e, mais tarde, em sua qualidade de vida; portanto, você deve avaliar cuidadosamente o seu desenvolvimento psicomotor, de modo que a detecção precoce dos factores de risco susceptíveis de garantir intervenções de cuidados.

PALAVRAS-CHAVE: desenvolvimento infantil, desempenho psicomotor, peso ao nascer, baixo peso neonatal. 
ISSN 1794-9831

E-ISSN 2322-7028

Vol. 12 No. 2

Jul - Dic 2015

Cúcuta, Colombia

\section{INTRODUCCIÓN}

egún la Organización Mundial de la Salud (OMS), se denomina recién nacido con bajo peso al nacer a un niño o niña con peso menor a los 2.500 gramos al momento del nacimiento, independiente de su edad gestacional. Este indicador constituye un factor de riesgo que puede desencadenar problemas neurológicos, desarrollo psicomotor inadecuado, enfermedades crónicas en la adultez, pérdida de funciones vitales como la visión y audición, sepsis y aumento en la mortalidad neonatal, entre otros (1-5).

Dentro de la población de mayor riesgo se encuentran los niños prematuros, aunque también se incluyen los nacidos a término, con bajo peso al nacer asociado, generalmente, a problemas durante su crecimiento intrauterino (6).

Este problema está relacionado con, al menos, el $60 \%$ de las muertes infantiles en países como Estados Unidos (7). Aunado a lo anterior, existen implicaciones en el desarrollo psicomotor de estos niños, tales como la disminución de habilidades de forma continua durante toda la infancia, que proviene tanto de una alteración en la maduración de las estructuras nerviosas como de la ausencia de estimulación y del aprendizaje que el niño hace, en su desarrollo, para descubrirse a sí mismo y al mundo que le rodea (8).

Los avances científicos en el sector salud han reducido notoriamente la cantidad de muertes infantiles derivadas de un bajo peso al nacer, así como las incapacidades que experimenta este grupo poblacional. No obstante, todavía existe un pequeño porcentaje de niños que en el transcurso de su desarrollo padecen problemas de salud relacionados con este evento, los cuales reducen su calidad de vida e implican cuidados especiales durante todo su ciclo vital (7).

En virtud de lo anterior, se plantea esta revisión bibliográfica con el objetivo principal de identificar los factores de riesgo que causan el bajo peso al nacer; así mismo, se busca analizar el impacto de este evento en el desarrollo psicomotor y las principales acciones del personal de salud en el cuidado de este niño(a).

La selección de los documentos bibliográficos se realizó a través de la ubicación en bases de datos como ScienceDirect, SciELO, Dialnet, Pubmed y Proquest. Se hizo una búsqueda bibliográfica basada en las revistas, publicaciones nacionales indexadas y publicaciones internacionales indexadas y homologadas para Colombia a través de Publindex de Colciencias, utilizando los siguientes descriptores: bajo peso, bajo peso al nacer, muy bajo peso al nacer y desarrollo psicomotor.

Los registros obtenidos permitieron la revisión de 65 artículos y documentos, mediante diferentes combinaciones de los descriptores. Posteriormente, se seleccionaron los artículos de acuerdo a su actualización, su rigor metodológico y científico y su relevancia en torno a los temas a tratar, obteniendo un total de 50 artículos.

\section{Factores de riesgo identificados para el bajo peso al nacer}

El nacimiento de niños con bajo peso se ha convertido en un problema relevante, puesto que éstos desarrollan múltiples problemas, ya sea en el período perinatal, en la niñez o en la edad adulta.

Para abordar el tema del bajo peso al nacer se debe iniciar por analizar la situación desde la etapa de gestación, identificando todos aquellos factores maternos o prenatales que tienen un fuerte impacto en la presencia de este evento.

Dentro de los factores maternos de riesgo se han identificado, en estudios a nivel mundial realizados desde el siglo pasado, que la raza afroamericana, la pobreza, la edad materna, el hábito de fumar, los antecedentes obstétricos adversos, la malnutrición materna al inicio de la gestación, la baja ganancia de peso durante el embarazo y las enfermedades crónicas de la madre son determinantes en la aparición de este problema de salud infantil (9).

En Colombia, el Instituto Nacional de Salud (10), dentro del protocolo de vigilancia en salud pública para bajo peso al nacer, expone los factores de riesgo agrupados de la siguiente manera: factores sociodemográficos como la edad materna menor a 20 y entre los periodos de 35 a 40 años, nivel socioeconómico bajo, riesgos médicos anteriores, riesgos médicos actuales, cuidados prenatales inadecuados y riesgos ambientales y de conducta. 
Al respecto, Martínez et al. (11) en su estudio Factores maternos y bajo peso al nacer, Bahía Honda, realizado en 2011, identificó que existe una asociación importante entre la calidad de la salud materna y la problemática del bajo peso al nacer, surgiendo de ahí la necesidad de identificar y modificar dichos factores oportunamente. Esto se asocia con lo afirmado por Delgado et al. (12), en la investigación Repercusión de la desnutrición materna sobre el nacimiento de niños con bajo peso, realizada en 2012, en donde se evidencia que el antecedente obstétrico predominante fue la desnutrición materna.

A nivel nacional, Quino y Barreto (13), en el año 2015 en Boyacá, hallaron que el factor desnutrición de la madre, durante la etapa prenatal, afecta al feto en la etapa gestacional e influye en el desarrollo del neonato en diferentes etapas de su ciclo vital. Aquí se coincide con lo mencionado por Periquet et al. (14), en su estudio Algunos factores de riesgo asociados al bajo peso al nacer, realizado en 2014, en el cual se determinó que el bajo peso constituye un problema de salud que se acrecienta aún más cuando hay presencia de desnutrición.

Cabe aclarar que no se trata sólo de desnutrición materna que origine bajo peso al nacer -y por ende genere una complicación en el desarrollo psicomotor del neonato-, sino también de la situación nutricional de ese neonato con un bajo peso al momento de su nacimiento y el impacto que tendrá la misma en la evolución de su desarrollo psicomotor.

La desnutrición materna, entonces, no es el único factor de riesgo que pueda desencadenar un neonato con un bajo peso al momento de su nacimiento. Jorna et al. (15), en su estudio Comportamiento del bajo peso al nacer en el Policlínico Universitario Héroes del Moncada (2006 -2010), publicado en 2012, concluye que entre su grupo poblacional, la mayor cantidad de recién nacidos con bajo peso se presentó en madres con antecedentes patológicos de enfermedades crónicas no transmisibles antes de la gestación, así como en madres que durante la gestación presentaron sepsis vaginal, hipertensión arterial gravídica o anemia.

Otro factor íntimamente asociado al bajo peso al nacer, que desempeña un papel determinante en el desarrollo del infante, es la prematuridad. Inguanzo et al (16), en su estudio epidemiológico del año 2012 denominado Caracterización del desarrollo del pre término en el primer año de vida, informan que los partos pre términos presentaron una elevada frecuencia de bajo peso en el recién nacido y, además, comprobaron una adecuada recuperación nutricional de dichos niños en el primer año de vida, a pesar de ciertas dificultades encontradas en su alimentación.

De igual manera, un estudio realizado en Estados Unidos en el año 2014 por Cheng et al. (17), que buscaba determinar el efecto de la exposición a múltiples riesgos sociales en el retraso cognitivo a los 9 meses de edad, demostró una relación significativa entre la exposición acumulativa al riesgo social y el retraso cognitivo, mayormente mediada por el bajo peso al nacer, hallando, como otro factor asociado a este problema, el riesgo social, que implica en muchas ocasiones la ausencia de cuidados en la madre y su hijo durante el periodo pre y posnatal, los cuales podrían prevenir su aparición. Así mismo, este trabajo resalta la importancia de desarrollar programas que aborden el contexto social de los niños de Estados Unidos, de manera que se puedan mejorar sus trayectorias de desarrollo.

Ante esta evidencia, se considera que la única manera de prevenir alteraciones en el desarrollo psicomotor de los niños y/o patologías asociadas al bajo peso al nacer consiste en conocer y evaluar minuciosamente todos los factores de riesgo asociados a este evento, de manera que se disponga de registros estadísticos permanentes que aseguren la atención temprana en salud a este grupo poblacional, tal como lo afirma Barra y Alvarado (18) en su estudio Frecuencia de riesgo neurobiológico en recién nacidos, realizado en 2012.

\section{Impacto del bajo peso al nacer en el desarrollo psicomotor}

A pesar de las medidas preventivas que se puedan implementar para evitar un problema como el bajo peso al nacer, quedan siempre múltiples casos de recién nacidos que lo padecerán y que, si no reciben el tratamiento adecuado, presentarán múltiples problemas y complicaciones a lo largo de su ciclo vital, principalmente en el ámbito del desarrollo psicomotor.

El Fondo de las Naciones Unidas para la Infancia (UNICEF) (19), posterior a la evaluación de este problema y desde hace más de una década, ha demostrado que los niños y niñas con bajo peso al
ISSN 1794-9831

E-ISSN 2322-7028

Vol. 12 No. 2

Jul - Dic 2015

Cúcuta, Colombia 
ISSN 1794-9831

E-ISSN 2322-7028

Vol. 12 No. 2

Jul - Dic 2015

Cúcuta, Colombia nacer "tienen más probabilidades de morir durante los primeros meses o los primeros años. Los que sobreviven tienen disminuidas las funciones del sistema inmunológico y corren mayor riesgo de padecer posteriormente varias enfermedades, incluida la diabetes y diversas cardiopatías. Tienen también propensión a seguir malnutridos y a tener menores coeficientes de inteligencia, además de discapacidades cognoscitivas" (19).

Por lo anterior, se han adelantado estudios sobre la morbimortalidad en los recién nacidos con muy bajo peso al nacer, como el desarrollado por Genes et al. (20) en el año 2010, sobre la morbimortalidad en recién nacidos de muy bajo peso al nacer en una Unidad de Neonatología, en el cual se demuestra que la morbilidad más frecuente fue la enfermedad de la membrana hialina en 59,6\% con una letalidad del $36,6 \%$, que la mortalidad global del grupo fue de $32,8 \%$ y la causa de mortalidad más frecuente estuvo constituida por las infecciones, la prematurez y la hemorragia pulmonar.

Hernández et al. (21) en su estudio Morbilidad a los dos años de edad en niños con un peso al nacimiento menor de 1.500 g., realizado en el año 2005, registran como factores de mal pronóstico neurológico la microcefalia a los dos años de edad corregida y la aparición de alteraciones de la sustancia blanca en los hallazgos radiográficos. Además, expresa una elevada preocupación por los datos relacionados con el crecimiento y el desarrollo, puesto que al menos el $18 \%$ de los 188 pacientes no alcanzó el percentil 3 de peso a los dos años de edad corregida.

Un estudio realizado por Cruz et al. (22) entre 1991 y 2010, que buscaba analizar la mortalidad perinatal en nacidos con un peso menor de 1.000 gramos, demostró que las tasas de mortalidad en este grupo de nacidos fue mayor a otros grupos de estudio. Se pudo evidenciar que los recién nacidos de bajo o muy bajo peso al nacer presentan altas tasas de morbilidad y mortalidad, generalmente asociadas a defectos o problemas en su ámbito de desarrollo psicomotor. Diferentes estudios demuestran, además, la aparición de ciertas enfermedades como características del impacto del bajo peso al nacer en el desarrollo psicomotor.

También en el estudio realizado por Fernández et al. (23), en el año 2014, denominado Supervivencia y morbilidad en recién nacidos de muy bajo peso al nacer en una Red Neonatal Sudamericana, se pudo constatar que de los 8.234 recién nacidos entre 24 y 31 semanas de edad gestacional, el 30,8\% presentó retinopatía del prematuro, el 7,2 \% hemorragia intraventricular grave y el 4,6 \% leucomalacia periventricular. Estas patologías conllevan a la disminución del desarrollo psicomotor en los niños y, de hecho, a un ciclo de desarrollo muy deficiente hasta su adultez (24).

La prematuridad como factor de riesgo para el bajo peso al nacer, fue estudiada por Riechi et al. (25) en el año 2011, en su investigación titulada Impacto de parto prematuro y bajo peso al nacer en la cognición, la conducta y el aprendizaje de los niños en edad escolar. En este estudio se afirma que niños, niñas y adolescentes entre los seis y 15 años de edad, con antecedente de bajo peso al nacer y prematurez, muestran alteraciones funcionales cerebrales asociadas a trastornos cognitivo-conductuales y trastornos de aprendizaje. Esta información es corroborada por otros investigadores como Baños et al. (26) en 2013, para quienes la prematurez es un factor de riesgo que con mayor frecuencia conlleva a trastornos en el desarrollo de esta población y se agudiza aún más cuando se acompaña de bajo peso al nacer.

Por otra parte, Borkoski et al. (27), en un estudio retrospectivo sobre la evaluación del muy bajo peso al nacer $(<=1.500 \mathrm{~g})$ como indicador de riesgo para la hipoacusia neurosensorial, señalan que el porcentaje de niños con el diagnóstico de hipoacusia neurosensorial entre los recién nacidos con muy bajo peso al nacer es muy superior al esperado en la población general; todos los diagnosticados con esta hipoacusia fueron prematuros $\mathrm{y}$, además, presentaron uno o dos factores más de riesgos auditivos, asociados al muy bajo peso al nacer.

A nivel de crecimiento y desarrollo y medidas antropométricas, Durá et al. (28) en el año 2014, desarrollaron un trabajo descriptivo denominado Estudio evolutivo longitudinal (desde el nacimiento hasta los 8 años) de las variables antropométricas en una cohorte de recién nacidos de muy bajo peso, evidenciando que de los 170 recién nacidos objeto de estudio, el $87,1 \%$ presentó un crecimiento recuperador que permitió alcanzar una talla normal a los 4 años de edad, pero a los 8 años el $8,9 \%$ de 
ese grupo no presentaba crecimiento recuperador, sin contemplarse la posibilidad de tratamiento con hormona de crecimiento.

Valdés et al. (29) en el año 2013, en un estudio que intentaba determinar las complicaciones metabólicas en 50 adolescentes con antecedentes de bajo peso al nacer, halló variables bioquímicas afectadas tales como la hipertrigliceridemia (más elevada en el sexo femenino) y la hiperglucemia y la hiperinsulinemia, que fueron más elevadas en el sexo masculino.

Con lo anterior concuerda García et al. (30) en su estudio El bajo peso al nacer es un factor de riesgo de diabetes mellitus tipo 1, desarrollado en el año 2009, en donde se observa que el menor índice de masa corporal (IMC) al nacer de los 100 pacientes con diabetes mellitus estudiados y el incremento de peso que se produce en los primeros dos años de vida en esta población, podría involucrar algunos aspectos durante la vida fetal, tales como el factor de riesgo de diabetes mellitus tipo 1 .

De igual manera, Krauel et al. (31), en su estudio Restricción posnatal del crecimiento en recién nacidos españoles de muy bajo peso con edad gestacional menor o igual a 32 semanas, realizado en 2008, destaca el hecho de que los recién nacidos de muy bajo peso al nacer, de no más de 32 semanas de gestación, durante su estancia en las unidades de cuidados intensivos de neonatología sufren una restricción de crecimiento respecto al patrón de crecimiento intrauterino. Dicha restricción ocurre sobre todo en los primeros 28 días de vida.

Además, según García et al. (32), en un estudio realizado en el año 2012 sobre las alteraciones neuropsicológicas y emocionales en prematuros con bajo peso al momento de su nacimiento, se evidenció que la presencia de la prematuridad, sumada al bajo peso, desencadenó alteraciones neuropsicológicas principalmente en la memoria y la función ejecutiva, así como algunos problemas de conducta y atención.

$\mathrm{Y}$ en otros ámbitos diferentes a parámetros antropométricos, García et al. (33), mencionan en su artículo sobre algunos factores de riesgo en la mortalidad de los recién nacidos de muy bajo peso con membrana hialina, que el incremento de peso en el neonato, así como el test de Apgar a los 5 minutos y la administración de agente tensión activo y corticoides, así como un grado leve de membrana hialina, disminuye la mortalidad en este grupo poblacional.

García et al. (34), en un estudio desarrollado en 2014 sobre factores de riesgo de hipotermia al ingreso en el recién nacido de muy bajo peso y morbimortalidad asociada, descubrieron que la proporción de recién nacidos de muy bajo peso al nacer y/o con 30 semanas de edad gestacional que ingresan con hipotermia es elevada en el ámbito de los servicios de salud. Así mismo, el bajo peso al nacimiento, el parto vaginal y la reanimación cardiopulmonar (RCP) avanzada fueron las principales variables relacionadas con la hipotermia y ésta, a su vez, se asoció con mayor riesgo de hemorragia intracraneal y de mortalidad.

De igual manera, Fanaroff et al. (35), en el año 2006, en su estudio La hipotensión tratada se asocia con morbilidad neonatal y déficit auditivo en los niños con peso al nacer extremadamente bajo, afirman que el tratamiento de la hipotensión durante las primeras 72 horas de vida del recién nacido con extremadamente bajo peso, se asocia con una morbilidad significativa a corto y largo plazo, con grandes posibilidades de retraso en el desarrollo motor, déficit auditivo y fallecimiento. Concluyen que es de vital importancia no abarcar solamente el aspecto biológico sino abordar al individuo con antecedente de bajo peso al nacer en todas sus esferas, como por ejemplo la intelectual.

Duus y Bircow (36), en un estudio comparativo sobre la calidad de vida, los logros académicos y el funcionamiento social de adultos jóvenes con bajo peso al nacer, demostraron que el grupo de bajo peso al nacer presentó una tasa más alta de deserción escolar, pero un funcionamiento social y una calidad de vida comparable a las del grupo con peso normal al nacer.

El impacto que tiene el bajo peso al momento del nacimiento en el futuro del niño y su desarrollo psicomotor y en otros múltiples aspectos de su ciclo vital, se pone de manifiesto con Jiménez et al. (37), quienes en su estudio Seguimiento de recién nacidos de peso menor o igual a $1.000 \mathrm{~g}$. durante los tres primeros años de vida, desarrollado en 2008 , resaltan que se presenta un aumento importante de la incidencia y supervivencia de recién nacidos de extremo bajo peso al nacer, quienes presentan un
ISSN 1794-9831

E-ISSN 2322-7028

Vol. 12 No. 2

Jul - Dic 2015

Cúcuta, Colombia 
ISSN 1794-9831

E-ISSN 2322-7028

Vol. 12 No. 2

Jul - Dic 2015

Cúcuta, Colombia mayor riesgo de secuelas en su desarrollo: retraso en la curva ponderal, parálisis cerebral, alteraciones oftalmológicas y auditivas y, en algunos casos, algún tipo de déficit en su desarrollo psicomotor o en su lenguaje.

El impacto causado por el bajo peso al nacer se evidencia, por lo tanto, en repercusiones de tipo neurológico que influyen potencialmente en el desarrollo psicomotor, afectando tanto su componente físico como psicosocial y del lenguaje (38-40).

Acciones del personal de salud en el cuidado del niño de bajo peso al nacer con afectación de su desarrollo psicomotor

A pesar de todas las complicaciones del bajo peso al nacer anteriormente mencionadas, siempre existe una esperanza para aportar a las familias afectadas. Así lo refiere Olds (41) en su ensayo del año 2006 denominado Progreso de la mejora del desarrollo de los neonatos de bajo peso al nacer, quien ratifica que se consigue algo verdaderamente importante para los neonatos vulnerables nacidos con un peso entre los 2.000 y 2.500 gramos cuando se identifica el riesgo y se realizan acciones oportunas para intervenirlo. Este autor invita a que se realice algo aún más importante por aquellos neonatos de menor peso y que estas intervenciones se realicen al menor coste posible.

En el mismo sentido, Pallás y el Grupo PrevInfad/ PAPPS Infancia y Adolescencia (42), en su revisión literaria denominada Actividades preventivas y de promoción de la salud para niños prematuros con una edad gestacional menor de 32 semanas o un peso inferior a $1.500 \mathrm{~g}$. del alta hospitalaria a los siete años ( $2^{\mathrm{a}}$ parte), alude que el riesgo de problemas en el desarrollo es inversamente proporcional a la edad gestacional. Es por esto que, para los niños prematuros con un bajo peso al momento de su nacimiento, se deben realizar cuidados preventivos y tratar tanto a los niños como a sus familias, de manera que se pueda mejorar su pronóstico.

Al respecto, Robaina y Riesgo (43) proponen un programa de seguimiento de recién nacidos de alto riesgo en Cuba, aseverando que la creación de tal programa de seguimiento no solamente cumple con el principio ético de justicia, sino que permitirá el restablecimiento de comparaciones de calidad de los servicios entre los centros de atención perinatal.
Por su parte, Valls et al. (44), en su estudio Morbimortalidad en recién nacidos de muy bajo peso en el control de la calidad de la asistencia perinatal, evidenciaron que la asistencia perinatal prestada a cada paciente con altos estándares de calidad permitió disminuir las tasas de morbilidad neonatal durante el período estudiado. Es aquí donde se destaca el sentido proactivo y multifuncional de la enfermería, con el propósito de capacitar en su cargo a cada persona y hacer de su servicio el más alto en la calidad de la atención.

De igual manera, la enfermera puede crear un programa de seguimiento al desarrollo de recién nacidos con bajo peso al nacer que sea aplicado tanto en instancia hospitalaria como a nivel ambulatorio. Por ejemplo, en España, en el año 2009, Agut et al. (45) valoraron la calidad de un programa de seguimiento del neurodesarrollo de prematuros de muy bajo peso al nacer, el cual fue manejado a través de visitas ambulatorias y demostró ser una herramienta muy útil para evaluar el progreso o retraso en el desarrollo de este grupo de pacientes.

La estimulación y el contacto madre-hijo precoz es también una manera ampliamente utilizada para ayudar a los niños con bajo peso al nacer y posible afectación de su desarrollo psicomotor. Lo anterior se ratifica en el estudio de Muñoz et al. (46), en donde se analizaron las interacciones de 31 madres y sus niños con diagnóstico de daño neurológico perinatal con bajo peso al nacer. Se demostró que en dichos niños las interacciones madre-hijo representan un buen predictor del desarrollo motor en el primer año de vida, lo cual es de gran importancia ya que los logros motores en los primeros meses representan un indicador predictivo del desarrollo infantil posterior.

En contraste con lo anterior, Rodríguez et al. (47), en un estudio retrospectivo realizado en 2011, indaga sobre la necesidad de la estimulación precoz en los recién nacidos con peso al nacer entre 1.000 y 1.500 g. Allí se evaluó a 194 recién nacidos y encontraron que quienes presentaban exploración neurológica normal al alta y Score pronóstico menor de 4 puntos, de acuerdo al rango establecido por ellos a pesar de su peso, no precisaban estimulación precoz desde el punto de vista neurológico, dada su previsible buena evolución.

Otra práctica implementada por el personal de enfermería es estudiada por Uko et al. (48), en su 
investigación Profilaxis a corto plazo con fluconazol en los niños con muy bajo peso al nacer y con peso al nacer extremadamente bajo, en donde aclara que la infección micótica invasiva (IMI) es común en los niños con muy bajo o extremadamente bajo peso al nacer y afirma que la profilaxis dirigida con fluconazol (sólo durante los períodos mayores a 3 días de administración de antibiótico y limitada a los niños con baja edad gestacional y bajo peso al nacer), puede ser tan eficaz como la profilaxis con fluconazol durante 4 a 6 semanas, para reducir el riesgo de IMI en este grupo poblacional. Además, disminuye el riesgo de desarrollar resistencia al antimicótico en los mismos.

De otro lado, un estudio realizado en Wisconsin (Estados Unidos), en el año 2012, por McManus et al. (49), determinó la efectividad de la intervención temprana física, ocupacional y terapia del habla en los servicios para prematuros o de bajo peso al nacimiento. Explicó, adicionalmente, que la intervención temprana se basó en medir la función cognitiva y el apoyo materno, con lo cual concluyó que la participación en la terapia de intervención temprana puede estar asociada con más óptimas trayectorias de la función cognitiva entre los niños prematuros cuyas madres tienen más y mejores apoyos, lo cual, al recibir dicha terapia, se torna particularmente beneficioso.

Por su parte, en la atención del recién nacido en la unidad de cuidados intensivos neonatales (UCIN), Ehrenkranz et al. (50), en 2006, en su estudio llamado El crecimiento en la UCIN influye sobre la evolución del neurodesarrollo y del crecimiento en los niños con peso al nacer extremadamente bajo, sugieren que la velocidad de crecimiento durante la hospitalización en la UCIN de un recién nacido con bajo peso, ejerce un significativo esfuerzo sobre las evoluciones del neurodesarrollo y del crecimiento de los 18 a los 22 meses de edad corregida, con lo cual se recalca la importancia del papel de enfermería en la administración idónea de la nutrición, ya sea de tipo enteral o parenteral, según las necesidades de cada recién nacido.

\section{CONCLUSIONES}

El bajo peso al nacer y su impacto en el desarrollo psicomotor están mediados por factores determinantes que inciden en la mayor afectación de los niños y sus familias. Se plantea que, prioritariamente, se deben identificar y prevenir oportunamente estos riesgos para evitar la aparición del evento que se convierte en desencadenante de problemas de salud para el niño o niña y que conlleva a altos costos sociales y económicos para la familia.

Se deben, por tanto, conocer y evaluar minuciosamente los problemas asociados al bajo peso al nacer, de manera que se pueda diseñar un plan de intervención para promover una mejor calidad de vida, centrado en el desarrollo saludable del niño y la niña.

El impacto que tiene el bajo peso al momento del nacimiento se evidencia en secuelas como el retraso en la curva ponderal, la parálisis cerebral, las alteraciones oftalmológicas y auditivas y, en algunos casos, el retraso en los alcances del desarrollo psicomotor, los cuales pueden ser intervenidos previniendo su aparición antes del nacimiento del niño y niña o a partir del seguimiento y evaluación permanente de este grupo poblacional.

Todo el equipo de salud no solamente debe brindar cuidado o actuar en los casos de niños con bajo peso al nacer y un desarrollo psicomotor ya afectado, sino comenzar siempre desde la prevención de ese bajo peso $y$, por ende, del retraso en el desarrollo psicomotor. Para cumplir con dicha meta, es preciso orientar la investigación hacia el cuidado de los niños y madres con antecedentes de partos con bajo peso al nacer y afectación del desarrollo psicomotor. Enfermería debe abanderarse en este tema y diseñar programas de seguimiento que permitan medir el impacto de las intervenciones realizadas a largo plazo.

\section{CONFLICTO DE INTERESES}

Los autores declaran no tener ningún conflicto de intereses.
ISSN 1794-9831

E-ISSN 2322-7028

Vol. 12 No. 2

Jul - Dic 2015

Cúcuta, Colombia

$$
\text { intereses. }
$$




\section{REFERENCIAS BIBLIOGRÁFICAS}

1. Castro-Carrasco P, Barraza-Rodríguez P. Diferencias cerebrales en prematuros y su relación con el desarrollo de sus funciones cognitivas. Ter Psicol [Internet]. 2007 [consultado 29 de abril de 2015]; 25(2): 183-188. Disponible en: http://www.scielo.cl/scielo.php?pid=S0718-48082007000200009\&script=sci_arttext

2. Souto S, Santos FDD, Coca L. Nacimiento de recién nacidos de bajo peso en institución filantrópica terciaria del Municipio de Piracicaba. Enferm. Glob. [Internet]. 2011 [consultado 29 de abril de 2015]; 10(23):61-75. Disponible en: http://scielo.isciii.es/scielo.php?pid=S1695-61412011000300006\&script=sci_arttext

3. Goide-Linares E, Rodríguez-Palomo A, Maturel-Zamora E, Palomo-Vidal B, Nillar-Vázquez Y. Factores de riesgo de sepsis en menores de un año. MEDISAN [Internet]. 2013 [consultado 29 de abril de 2015]; 17(6): 915919. Disponible: $\mathrm{http}: / /$ scielo.sld.cu/scielo.php?pid=S1029-30192013000600005\&script=sci_arttext

4. Moreno-Mora R, Pérez-Díaz C. Retardo en el neurodesarrollo en niños de La Habana Vieja. Rev Cubana Med Gen Integr [Internet]. 2008 [consultado 29 de abril de 2015]; 24(3). Disponible en: http://scielo.sld.cu/scielo. php?pid=S0864-21252008000300012\&script $=$ sci_arttext

5. Chueca MJ, Berrade S, Ovarzábal M. Talla baja y enfermedades raras. Anales Sis San Navarra [Internet]. 2008 [consultado 29 de abril de 2015]; 31(2). Disponible en: http://scielo.isciii.es/scielo.php?script=sci_arttext\&pid $=$ S1137-66272008000400004

6. Ecured. Bajo peso al nacer. [Internet]. Cuba 2007 [consultado 28 de abril de 2015]. Disponible en: http://www. ecured.cu/index.php/Bajo_peso_al_nacer

7. GeoSalud. Bajo peso al nacer. [Internet]. San José, Costa Rica [consultado 29 de abril de 2015]. Disponible en: http://www.geosalud.com/embarazo/bajo_peso_al_nacer.htm

8. Clínica Universidad de Navarra. Desarrollo psicomotor del niño. [Internet]. España; 2015 [consultado 29 de abril de 2015]. Disponible en: http://www.cun.es/chequeos-salud/infancia/desarrollo-psicomotor

9. Rauh VA, Howard A, Robin G. The Contribution of maternal age to racial disparities in birthweigth: a multilevel perspective. Am J Public Health. [Internet]. 2001 [consultado 29 de abril de 2015]; 91(11): 1815-1824. Disponible en: http://www.ncbi.nlm.nih.gov/pmc/articles/PMC1446885/

10. Instituto Nacional de Salud - INS. Protocolo de vigilancia en salud pública: bajo peso al nacer. [Internet]. 2014 [consultado 2 de noviembre de 2015]. Disponible en: http://www.ins.gov.co/lineas-de-accion/SubdireccionVigilancia/sivigila/Protocolos\%20SIVIGILA/PRO\%20Bajo\%20Peso\%20al\%20Nacer\%20a\%20Termino.pdf

11. Martínez-Argudín XE, Duarte-Duarte Y, Portales-Gil E, Mirabal- Martínez G. Factores maternos y bajo peso al nacer, Bahía Honda. Rev Ciencias Médicas [Internet]. 2011 [consultado 29 de abril de 2015]; 15(1):89-102. Disponible en: http://scielo.sld.cu/scielo.php?script=sci_arttext\&pid=S1561-31942011000100008

12. Delgado-Álvarez I, Roca-Rosales MC, Suárez-Vega M, Rodríguez-Alarcón JD, Ruíz-Echavarría Y. Repercusión de la desnutrición materna sobre el nacimiento de niños con bajo peso. Medisan [Internet]. 2012 [consultado 29 de abril de 2015]; 16(10):1478-1485. Disponible en: http://scielo.sld.cu/scielo.php?pid=S102930192012001000002\&script $=$ sci_arttext

13. Quino AC, Barreto P. Desarrollo motor en niños con desnutrición en Tunja, Boyacá [Internet]. 2015 [consultado 29 de abril de 2015]; 33(1):15-21. Disponible en: http://www.scielo.org.co/scielo.php?pid=S0120386X2015000100003\&script=sci_arttext\&tlng=es

14. Periquet-Meriño M, Pascao-Gamboa A, Labaut-Ginarte O, Vargas-de La Paz L, Mora-Nieto J. Algunos factores de riesgo asociados al bajo peso al nacer en el Hospital General "Orlando Pantoja Tamayo". Medisan [Internet]. 2014 [consultado 29 de abril de 2015]; 18(1):11-16. Disponible en: http://scielo.sld.cu/scielo.php?script=sci_art text\&pid=S1029-30192014000100003

15. Jorna-Calixto AR, Rodríguez-Crespo I, Véliz-Martínez PL, Cuellar-Álvarez R, Olivera-Nodarse Y. Comportamiento del bajo peso al nacer en el Policlínico Universitario "Héroes del Moncada (2006-2010). Rev Cubana de Med Gen Integr [Internet]. 2012 [consultado 29 de abril de 2015]; 28(1): 3-14. Disponible en: http:// scielo.sld.cu/scielo.php?script=sci_arttext\&pid=S0864-21252012000100002

16. Inguanzo-Clausell $\mathrm{O}$, Valdés-Abreu $\mathrm{BM}$, Iturriagagoitia-Herrera $\mathrm{E}$, García-Rodríguez L, Hernández-Hierrezuelo ME. Caracterización del desarrollo dl pretérmino en el primer año de vida. Rev. Ciencias Médicas [Internet]. 2012 [consultado 29 de abril de 2015]; 16(1): 146-157. Disponible en: http://scielo.sld.cu/scielo.php?script=sci_ arttext\&pid=S1561-31942012000100016

17. Cheng ER, Poehlmann-Tynan J, Mullahy J, Witt WP. Cumulative social risk exposure, infant birth weight, and cognitive delay in infancy. Academic Pediatrics [Internet]. 2014 [consultado 29 de abril de 2015]; 14(6): 581588. Disponible en: http://www.academicpedsjnl.net/article/S1876-2859(14)00097-7/pdf

18. Barra L, Alvarado R. Frecuencias de riesgo neurológico en recién nacidos. Rev chil pediatr [Internet]. 2012 
Bajo peso al nacer y su implicación en el desarrollo psicomotor • Doris Amparo Parada-Rico, Nathalia López-Guerrero, Marcela Martínez-Laverde.

[consultado 29 de abril de 2015]; 83 (6): 552-562. Disponible en: http://webcache.googleusercontent.com/ search?q=cache:http://www.scielo.cl/pdf/rcp/v83n6/art04.pdf

19. Fondo de la Naciones Unidas para la Infancia - UNICEF. Bajo peso al nacer. [Internet] 2001. [consultado 2 de noviembre de 2015]. Disponible en: http://www.unicef.org/spanish/specialsession/about/sgreport-pdf/15 LowBirthweight_D7341Insert_Spanish.pdf

20. Genes L, Lacarrubba L, Caballero C, Fonseca R, Mir R, Céspedes E, et al. Morbi-mortalidad en recién nacidos de muy bajo peso al nacer. Unidad de Neonatología. Centro Materno Infantil. Hospital de Clínicas. Pediatr: (Asunción) [Internet]. 2010 [consultado 30 de abril de 2015]; 37(1):9-22. Disponible en: http://dialnet.unirioja. es/servlet/articulo? codigo $=4800234$

21. Hernández-González N, Salas-Hernández S, García-Alix Pérez A, Roche-Guerrero C, Pérez-Rodríguez $\mathrm{J}$, Omeñaca-Teres $\mathrm{F}$, et al. Morbilidad a los dos años de edad en niños con un peso al nacimiento menor de 1.500 g. An Pediatr [Internet]. 2005 [consultado 29 de abril de 2015]; 62(4):320-7. Disponible en: http://www. analesdepediatria.org/es/morbilidad-los-dos-anos-edad/articulo/13073244/

22. Cruz-Martínez M, Redondo-Aguilar R, Caño-Aguilar A, Carretro-Lucena $P$, Padilla-Vinuesa C, Ruiz-Extremera A. Análisis de la mortalidad perinatal en nacidos con peso menor de 1000 gramos en el Hospital San Cecilio de Granada (España), durante el período 1991 - 2010. Arch argent pediatr [Internet]. 2013 [consultado 29 de abril de 2015];111(1):45-52. Disponible en: http://www.scielo.org.ar/scielo.php?script=sci_arttext\&pid=S032500752013000100009\&lng=es\&nrm=iso\&tlng=es

23. Fernández R, D’Apremont I, Domínguez A, Tapia JL, Red Neonatal Neocosur. Supervivencia y morbilidad en recién nacidos de muy bajo peso al nacer en una Red Neonatal Sudamericana. Archivos Argentinos de Pediatría [Internet]. 2014 [consultado 29 de abril de 2015];112(5): 405-412. Disponible en: http://www.sap.org.ar/docs/ publicaciones/primero/2014/AO_Fernandez_Anticipo_1-8-14.pdf

24. Rivas-Ruíz R, Guzmán-Cabañas JM, Párraga MJ, Ruiz-González MD, Huertas-Muñoz MD, Álvarez-Marcos R. Utilidad del CRIB para predecir la muerte hospitalaria y la hemorragia intraventricular en los prematuros de muy bajo peso y extremado bajo peso al nacer. Anales de Pediatría [Internet]. 2007 [consultado 29 de abril de 2015]; 66(2):140-145. Disponible en: http://dialnet.unirioja.es/servlet/articulo?codigo=2249186

25. Riechi TI, Valeriana ML, Ciasca SM. Impacto de parto prematuro y bajo peso al nacer en la cognición, la conducta y el aprendizaje de los niños en edad escolar. Rev. paul. pediatr. [Internet]. 2011 [consultado 29 de abril de 2015]; 29(4):495-501. Disponible en: http://www.scielo.br/scielo.php?script=sci_arttext\&pid=S010305822011000400005\&lng=en\&nrm=iso\&tlng=en

26. Baños-Carmona OL, Toledo-González Y, Soto-García M, Mier-Armas M, Rúa-Martínez R, Lapido-Polanco S. Comportamiento de la retinopatía de la prematuridad en la provincia de La Habana. Revista Cubana de Oftalmología [Internet]. 2013 [consultado 29 de abril de 2015]; 26(2):294-306. Disponible en: http://scielo.sld. $\mathrm{cu} / \mathrm{pdf} / \mathrm{oft} / \mathrm{v} 26 \mathrm{n} 2 / \mathrm{oft} 12213 . \mathrm{pdf}$

27. Borkoski-Barreiro SA, Falcón-González JC, Liminaña-Cañal JM, Ramos-Macías A. Evaluación del muy bajo peso $(<=1.500 \mathrm{~g})$ al nacer como indicador de riesgo para la hipoacusia neurosensorial). Acta Acta Otorrinolaringol Esp. [Internet]. 2013 [consultado 29 de abril de 2015];64(6):403-408. Disponible en: http://www.elsevier.es/esrevista-acta-otorrinolaringologica-espanola-102-pdf-90252703-S300?login=ko

28. Durá-Trave T, San Martín-Garcia I, Chueca-Guinduláin MJ, Berrade-Zubiri S. Estudio evolutivo longitudinal (desde el nacimiento hasta los 8 años) de las variables antropométricas en una cohorte de recién nacidos de muy bajo peso. Nutrición Hospitalaria. Órgano Oficial de la Sociedad Española de Nutrición Parenteral y Enteral [Internet]. 2014 [consultado 30 de abril de 2015]; 30(5): 1063-1070. Disponible en: http://dialnet.unirioja.es/ servlet/articulo? codigo $=4862137$

29. Valdés-Alonso MC, Rodríguez-Suarez CA, Basain-Valdés JM, Piñeiro-Lamas CR, Ladrón de Guevara-Casals A. Algunas variables bioquímicas en adolescentes obesos con antecedente de bajo peso al nacer. Rev Cubana Pediatr [Internet]. 2013 [consultado 29 de abril de 2015]; 85(4):439-447. Disponible en: http://scielo.sld.cu/ scielo.php?pid=S0034-75312013000400004\&script=sci_arttext

30. García-Cuartero B, González-Vergáz A, Herranz S, Vásquez MC, Carvajal O, Carpintero P, et al. El bajo peso al nacer es un factor de riesgo de Diabetes Mellitus Tipo 1. An Pediatr [Internet]. 2009 [consultado 30 de abril de 2015];70:542-6. Disponible en: http://www.analesdepediatria.org/es/el-bajo-peso-al-nacer/articulo/ S169540330900215X/

31. Krauel-Vidal X, Figueras-Aloy J, Natal-Pujol A, Iglesias-Platas I, Moro-Serrano M, Fernández-Pérez E, et al. Restricción posnatal del crecimiento en recién nacidos españoles de muy bajo peso con edad gestacional menor o igual a 32 semanas. An Pediatr [Internet]. 2008 [consultado 30 de abril de 2015]; 68(3): 206-12. Disponible en: http://www.analesdepediatria.org/es/restriccion-posnatal-del-crecimiento-recien/articulo/S1695403308700596/ 
ISSN 1794-9831

E-ISSN 2322-7028

Vol. 12 No. 2

Jul - Dic 2015

Cúcuta, Colombia
32. García-Bermúdez O, Cruz-Quintana F, Sosa MA, De la Cruz J, Mañas M, Pérez-García M. Alteraciones neuropsicológicas y emocionales en niños de muy bajo peso al nacer. Revista Argentina de Ciencias del Comportamiento (RACC) [Internet]. 2012 [consultado 30 de abril de 2015]; 4(2):3-10. Disponible en: http:// dialnet.unirioja.es/servlet/articulo?codigo $=3983565$

33. García-Arias MB, Zuluaga-Arias P, Arrabal-Teran MC, Arizcun-Pineda J. Factores de riesgo en la mortalidad de los recién nacidos de muy bajo peso con membrana hialina. An Pediatr [Internet] 2005 [consultado 30 de abril de 2015]; 63(2): 109-15. Disponible en: http://www.analesdepediatria.org/es/factores-riesgo-mortalidad-los-recien/ articulo/13077451/

34. García-Muñoz F, Rivero-Rodríguez S, Siles-Quesada C. Factores de riesgo de hipotermia al ingreso en el recién nacido de muy bajo peso y morbimortalidad asociada. An Pediatr [Internet]. 2014 [consultado 30 de abril de 2015]; 80(3):144-50. Disponible en: http://www.analesdepediatria.org/es/factores-riesgo-hipotermia-al-ingreso/ articulo/S1695403313003482/

35. Fanaroff JM, Wil son D, Newman N, Mompetit M, Fanaroff A. La hipotensión tratada se asocia con morbilidad neonatal y déficit auditivo en los niños con peso al nacer extremadamente bajo. Pediatrics (Ed esp) [Internet]. 2006 [consultado 30 de abril de 2015];61(4): p. 278. Disponible en: http://apps.elsevier.es/watermark/ctl servlet? f=10\&pident_articulo $=13113570$ \&pident_usuario $=0$ \&pcontactid $=$ \&pident revista $=10 \&$ ty $=93 \&$ accio $\mathrm{n}=$ L\&origen=zonadelectura\&web=www.elsevier.es\&lan=es\&fichero=10v61n04a13113570pdf001.pdf

36. Duus-Odberg M, Bircow-Elgen I. Low birth weight young adults: quality of life, academic achievements and social functioning. Acta Paediátrica [Internet]. 2011 [consultado 30 de abril de 2015]; 100(2): 284-288. Disponible en: http://onlinelibrary.wiley.com/doi/10.1111/j.1651-2227.2010.02096.x/abstract

37. Jiménez-Martin AM, Servera-Ginard C, Roca-Jaume A, Frontera-Juan G, Pérez-Rodríguez J. Seguimiento de recién nacidos de peso menor o igual a $1.000 \mathrm{~g}$ durante los tres primeros años de vida. An Pediatr [Internet]. 2008 [consultado 30 de abril de 2015]; 68(4):320-8. Disponible en: http://www.analesdepediatria.org/es/seguimientorecien-nacidos-peso-menor/articulo/S1695403308700870/

38. Garófalo-Gómez N, Gómez-García AM, Vargas-Díaz J, Novoa-López L. Repercusión de la nutrición en el neurodesarrollo y la salud neuropsiquiátrica de niños y adolescentes. Rev Cubana Pediatr [Internet]. 2009 [consultado 29 de abril de 2015]; 81(2). Disponible en: http://scielo.sld.cu/scielo.php?pid=S0034$75312009000200008 \&$ script $=$ sci_arttext

39. Salas R, Sanhueza L, Maggi L. Factores de riesgo y seguimiento clínico en prematuros menores de 1000 gramos. Rev Chil Pediatr [Internet]. 2006 [consultado 29 de abril de 2015]; 77(6): 577-588. Disponible en: http://www. scielo.cl/scielo.php?script=sci_arttext\&pid=S0370-41062006000600004

40. Martínez JW. Desarrollo infantil: una revisión. Investig. andina [Internet]. 2014 [consultado 29 de abril de 2015]; 16(29): 1118-1137. Disponible en: http://www.scielo.org.co/scielo.php?pid=S0124$81462014000200010 \&$ script $=$ sci arttext

41. Olds D. Progreso de la mejora del desarrollo de los neonatos de bajo peso al nacer. Pediatrics (Ed esp) [Internet]. 2006 [consultado 30 de abril de 2015]; 61(3):146. Disponible en: http://apps.elsevier.es/watermark/ctl_servlet? $\mathrm{f}=10$ \&pident_articulo $=13113576 \&$ pident_usuario $=0$ \&pcontactid $=\&$ pident_revista $=10 \&$ ty $=99 \&$ accion $=$ L\&ori gen=zonadelectura \&web=www.elsevier.es\&lan=es\&fichero=10v61n03a13113576pdf001.pdf

42. Pallás-Alonso CR, Grupo PrevInfad/PAPPS Infancia y Adolescencia. Rev Pediatr Aten Primaria [Internet]. 2012 [consultado 29 de abril de 2015]; 14(55): 249-263. Disponible en: http://scielo.isciii.es/scielo.php?pid=S1139$76322012000400014 \&$ script $=$ sci_arttext

43. Robaina-Castellanos GR, Riesgo-Rodríguez SC. Propuesta de programa de seguimiento de recién nacidos de alto riesgo en Cuba. Rev Méd Electrón [Internet]. 2011 [consultado 29 de abril de 2015]; 33(5):645-655. Disponible en: http://scielo.sld.cu/scielo.php?pid=S1684-18242011000500012\&script $=$ sci_arttext

44. Valls-i Soler A, Páramo-Andrés S, Centeno-Monterubio C, Ansó-Oliván S, Gortazar-Arias P, López-Heredia I, et al. Morbimortalidad en recién nacidos de muy bajo peso en el control de la calidad de la asistencia perinatal. An Pediatr [Internet]. 2005 [consultado 30 de abril de 2015]; 58(3): 464-70. Disponible en: http://www. analesdepediatria.org/es/morbimortalidad-recien-nacidos-muy-bajo/articulo-resumen/S1695403303780941/

45. Agut-Quijano T, Conde-Cuevas N, Iriondo-Sanz M, Poo-Argüelles P, Ibañez-Fanes M, Krauel-Vidal X. Valoración de la calidad de un programa de seguimiento del neurodesarrollo de prematuros de muy bajo peso al nacimiento. An Pediatr [Internet]. 2009 [consultado 30 de abril de 2015]; 70(6): 534-41. Disponible en: http:// www.analesdepediatria.org/es/valoracion-calidad-un-programa-seguimiento/articulo/S169540330900246X/

46. Muñoz-Ledo Rabago $P$, Méndez-Ramírez I, Sánchez-Pérez C, Mandujano-Valdés M, Murata C. Interacciones tempranas madre-niño y predicción de desarrollo motor mediante ecuaciones estructurales: Aplicación del modelo en niños con riesgo de daño neurológico perinatal. Interdisciplinaria [Internet]. 2013 [consultado 29 
de abril de 2015]; 30(1): 119-138. Disponible en: http://www.scielo.org.ar/scielo.php?script=sci_arttext\&pid $=\mathrm{S} 1668-70272013000100007$

47. Rodríguez-Ogando A, Miranda-Herrero MC, Castro-Castro P, Vásquez-López M, Blando-Bravo D. Estimulación precoz en los recién nacidos con peso al nacer entre 1.000 y $1.500 \mathrm{~g}$. ¿Es siempre necesaria? An Pediatr An Pediatr [Internet]. 2011 [consultado 30 de abril de 2015]; 75(3):161-8. Disponible en: http://www.analesdepediatria.org/ es/estimulacion-precoz-los-recien-nacidos/articulo/S1695403310005540/

48. Uko S, Soghier LM, Vega M, Marsh J, Reinersman GT, Herring L, et al. Profilaxis a corto plazo dirigida con Fluconazol en los niños con muy bajo peso al nacer y con peso al nacer extremadamente bajo. Pediatrics (Ed esp) [Internet]. 2006 [consultado 30 de abril de 2015]; 61(4): p. 283. Disponible en: http://apps.elsevier.es/watermark/ ctl_servlet?_f $=10 \&$ pident_articulo $=13113570 \&$ pident_usuario $=0 \&$ pcontactid $=\&$ pident_revista $=10 \&$ ty $=93 \&$ ac cion $=$ L\&origen $=$ zonadelectura\&web=www.elsevier.es\&lan $=$ es\&fichero $=10 \mathrm{v} 61 \mathrm{n} 04 \mathrm{a} 13113570 \mathrm{pdf001} . \mathrm{pdf}$

49. McManus B, Carle AC, Poehlmann J. Effectiveness of part $C$ early intervention physical, occupational, and speech therapy services for preterm or low birth weight infants in Wisconsin, United States. Academic Pediatrics [Internet]. 2012 [consultado 30 de abril de 2015]; 12(2):96-103. Disponible en: http://www.academicpedsjnl.net/ article/S1876-2859(11)00290-7/fulltext

50. Ehrenkranz RA, Dusick Am, Vohr BR, Wright L, Wrage L, Poole WK. El crecimiento en la unidad de cuidados intensivos neonatales influye sobre la evolución del neurodesarrollo y del crecimiento en los niños con peso al nacer extremadamente bajo. Pediatrics (Ed esp) [Internet]. 2006 [consultado 29 de abril de 2015]; 61(4): p. 284. Disponible en: http://apps.elsevier.es/watermark/ctl_servlet?_f=10\&pident_articulo=13113570\&pident_ usuario $=0 \&$ pcontactid $=\&$ pident_revista $=10 \&$ ty $=93 \&$ accion $=$ L\&origen $=$ zonadelectura $\& w e b=w w w . e l s e v i e r . e s$ \&lan $=$ es\&fichero $=10 \mathrm{v} 61 \mathrm{n} 04 \mathrm{a} 13113570 \mathrm{pdf001} . \mathrm{pdf}$
ISSN 1794-9831

E-ISSN 2322-7028

Vol. 12 No. 2

Jul - Dic 2015

Cúcuta, Colombia 